\title{
Optogenetics and its influence on the clinical neurosciences
}

\author{
Mateusz Gotowiec*1 \\ ${ }^{1}$ UCL Medical School \\ *Correspondence to: Mateusz Gotowiec, mateusz.gotowiec.19@ucl.ac.uk
}

In 1979, Francis Crick proposed an innovative solution: the use of light to control neurons [1]. His idea, combined with the prolonged effort of various scientists, resulted in a revolutionary technique - optogenetics - being chosen in 2010 as the "Method of the Year" across all branches of science and engineering [2,3]. The first optogenetic experiments commenced in 2005, however, it took several years until this novel method began to be used in clinical neuroscience. In 2013, the European Brain Research Prize was awarded to Ernst Bamberg, Edward Boyden, Karl Deisseroth, Peter Hegemann, Gero Miesenböck, and Georg Nagel for their 'invention and refinement of optogenetics' [4]. Using optogenetics, scientists could for the first time examine the spatial, temporal, and cell-type resolution of the nervous system with such levels of precision.

This paper aims to highlight three major improvements in neuroscience which occurred following optogenetics development. Firstly, a possibility was created for singular cell specificity activation and the identification of particular neuronal networks, which led to a better understanding of neurodegenerative processes in conditions such as Parkinson's disease [5]. Secondly, optogenetics introduced a new approach to treatment, underlining the need for a shift from molecular-centric to pathway-centric concepts. Finally, it began a substantial revolution in neuroscience, which is currently significantly influencing other branches of science such as biotechnology and neurotechnology.

This literature review includes publications searched using PubMed and Web of Science databases based on a variety of keywords ('optogenetics', 'opsins', 'opsin', 'optogenetic', 'opto'). As the development is quite recent, only sources between 2005 and 2019 have been used with the majority having been published in the last decade. Only English language literature was used, including a great portion of research papers showing optogenetic experiment methodology, with lesser evidential value and a few review papers focused on optogenetics applicability.

\section{Optogenetics - the basics of its action}

Optogenetics is a unique domain in the complex of experimental and therapeutic approaches in the field of neurobiology because it creates a possibility for the manipulation of normal and pathological neuronal networks, which cannot be achieved using any other techniques. The underlying idea behind this biological technique is the control of neurons using light, primarily with photosensitive channels or pumps [6] which by changing ionic concentrations can depolarise or hyperpolarise the whole cell. The aforementioned neuronal control is achieved through optogenetic actuators (opsins) such as channelrhodopsin, halorhodopsin, and archaerhodopsin, while the recording of any changes caused by these proteins can be done using optogenetic sensors of several types, including calcium or voltage change detectors.

Opsins are a group of light-sensitive proteins (ion channels or G-protein coupled receptors) which can interfere with cell membrane potential in response to light stimuli [6]. The main class of opsins used for the activation of neurons are type I opsins, also known as microbial. They are preferentially employed as they allow a direct membrane potential change, leading to an action potential transduction, thus having an immediate effect [7]. The second class used, type II opsins, also called animal opsins, are a group of the G protein-coupled receptor (GPCR) superfamily. As opposed to type I opsins, they act indirectly by changing the metabolic pathways in the neuron [8], thus not provoking an instant response. The first step for optogenetic procedures is injecting benign viruses, most commonly adeno-associated viruses (AAV) [9] containing opsin-coding genes into a selected group of neurons. In this way, the neural cells become sensitive to light. Proteins, which act as switches, activate or disactivate the neurons depending on light flashes which are sent in milliseconds through fiber optic cables. Hence, each neuron can be depolarized or hyperpolarized using only the choice of wavelength that it receives. In this manner, the manipula- 


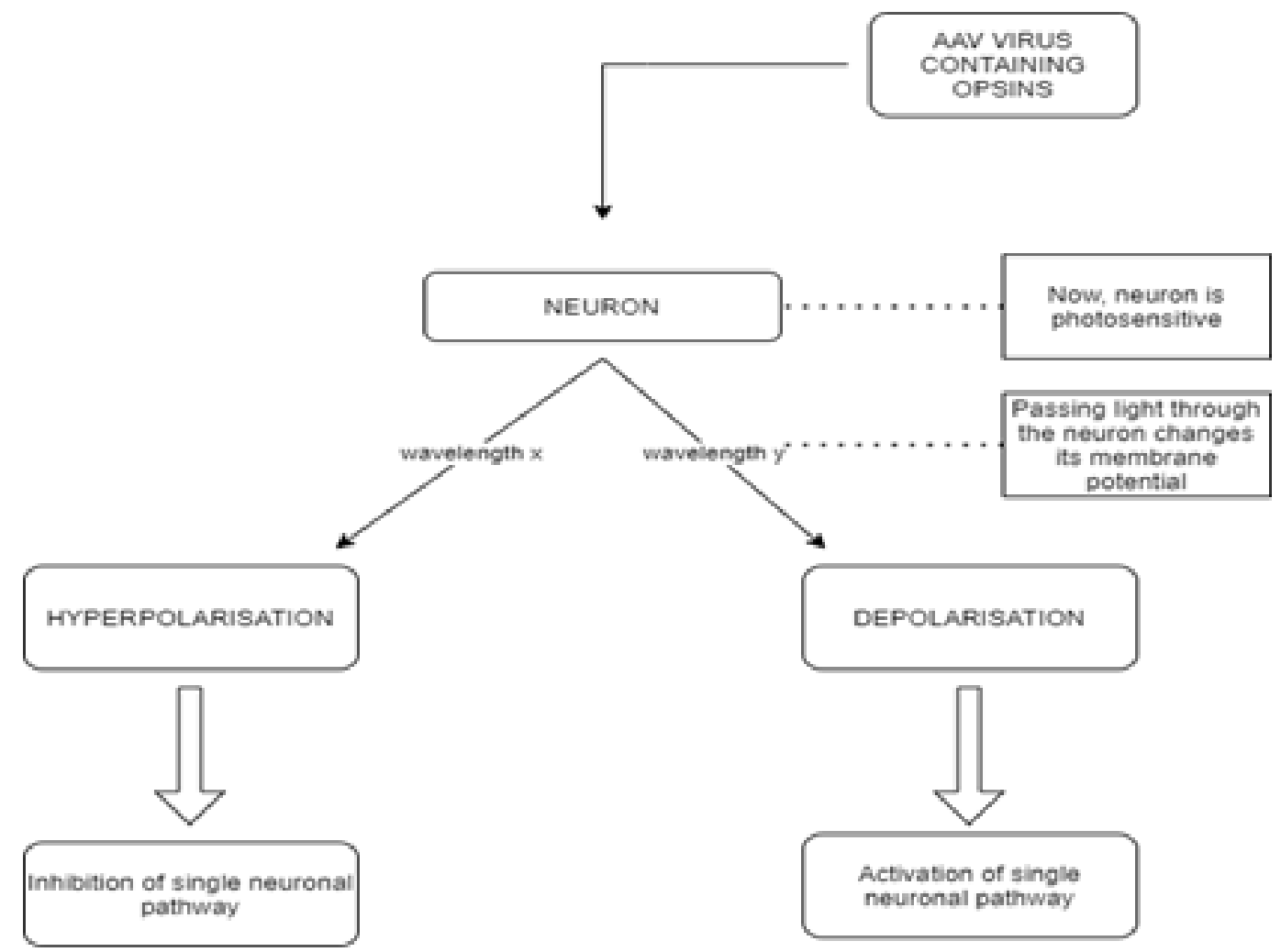

Figure 1. Chart showing basic optogenetic method.

tion of singular neuronal activities becomes possible for researchers.

\subsection{Control over electrical and biochemical activ- ity}

Optogenetics have not only allowed for the control of electrical activity, but also of many biochemical processes. As stated previously, there are two types of opsins, which allow both electrical and biochemical manipulation of the neurons. Most commonly, researchers focus on changing the electrical pattern of neuronal network activation, however, there is also a significant number of studies focused on manipulating the metabolic activity of cells. OptoXRs are a new family of receptors sensitive to green light, made from a combination of GPCR membrane receptors (which normally respond to many medicinal drugs) and the rhodopsin domain [10]. When introduced into a mouse via a virus vector, optoXRs respond to light and successfully exercise certain biochemical transduction mechanisms.

Furthermore, not only signal transduction can be influenced by optogenetic protocols, but also cell motility. Cofilin is a member of the cofilin/actin-depolymerising factor family, which acts on the actin filaments of the cytoskeleton of a cell, regulating its motility. An optogenetic version of cofilin, optocofilin, can be directed to target areas of the cytoskeleton, allowing for the spatiotemporal control of motility of the cell in the direction of a localized light $[11,12]$. Similarly, scientists were able to trigger light-dependent cellular apoptosis. Using optogenetically manipulated Bcl-2 family proteins, responsible for mitochondrial outer membrane permeabilization (MOMP) [13], they were able to recruit $\mathrm{Bcl}-2$ proteins to the outer mitochondrial membrane (OMM) with a light trigger. A mutant cytosolic Bax protein was fused with a blue-light sensitive Cry2 protein, creating the optoBcl-2, which was then directed to the OMM using a light source. The subsequent light-dependent cellular collapse occurred in a cascade manner, with the mitochondrion-bound optoBcl-2 leading to pore formation in the OMM, the leakage of cytochrome $\mathrm{c}$, the activation of the caspase pathway and eventually to cell death [12]. Potentially, the control of cell metabolism through optoXRs, cell motility via optocofilin and apoptosis using optoBcl-2 may lead to future therapeutic innovations.

\subsection{Precise study of neural circuits providing a novel approach}

Single-cell electrical activation is an important change brought by optogenetics, as it has paved a new way for analyzing specific neural circuits. Thanks to this development, scientists were able to causally link neural circuits, behavior, and function in diseases such as Parkinson's syndrome [9]. As optogenetics enables the active modulation of neuronal activity with cell-type and anatomical specificity, it can provide a powerful extension tool in combination with electrophysiological or optical techniques [9]. As 


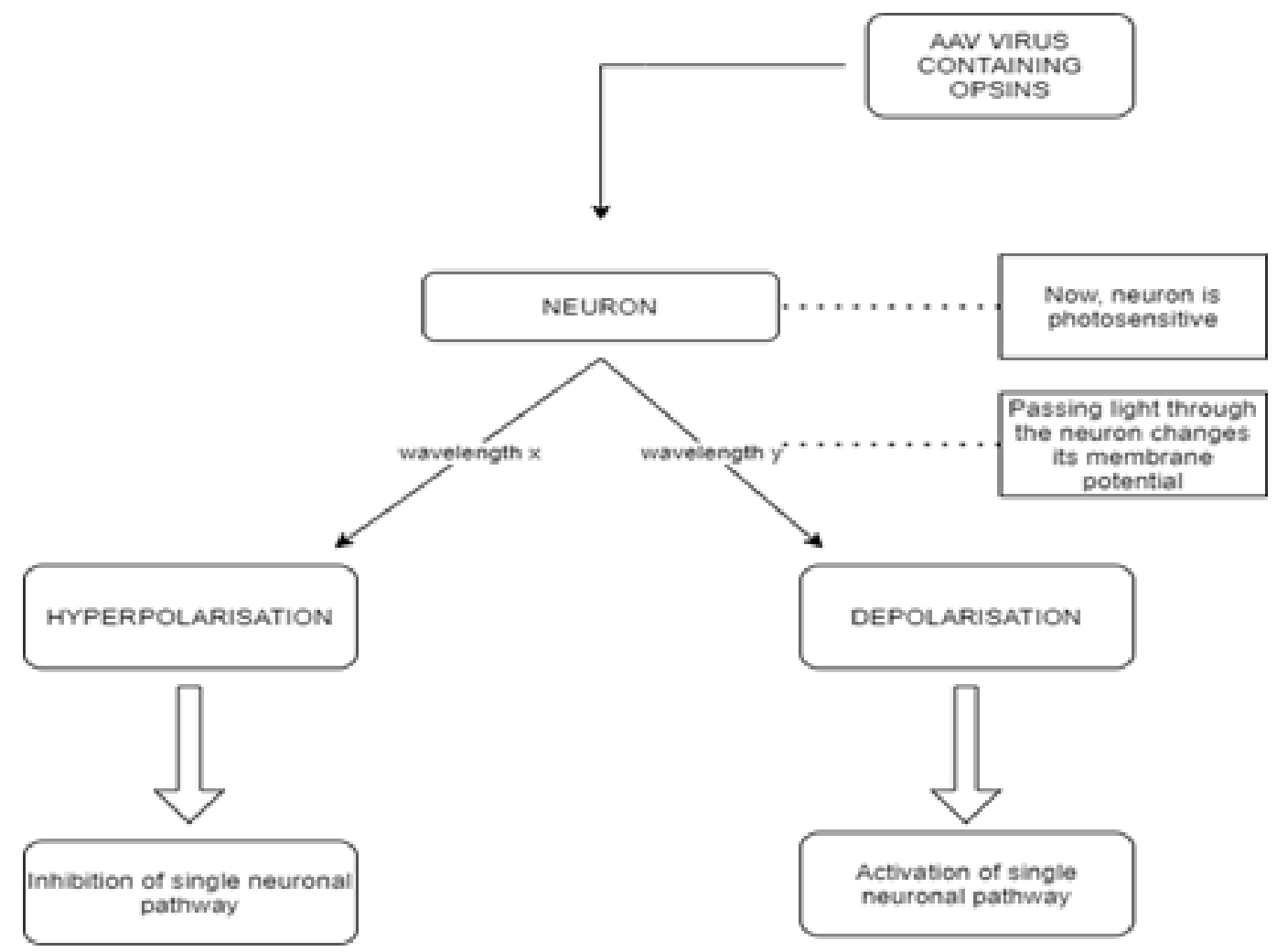

Figure 2. Chart showing developments made using optogenetics.

a result, an integrative understanding of neural circuitry can be achieved. Prior to optogenetics development, the most precise tools for investigating living, functioning brains, such as fMRI could only yield images of neuronal activity on a large scale [14]. Contrastingly, optogenetic methods allow the activity of single neural cells and the pathways which they constitute to be observed.

Comparing optogenetics to other methods used in clinical neurosciences, its selectivity and precision proves to be of a great advantage. Even the domain of pharmacogenetics, which developed shortly prior to optogenetics has not been able to achieve the precise spatiotemporal resolution of light-driven methods. On the other hand, despite different concepts both can yield similar effects, such as in the case of stimulation of AgRP neurons by optogenetic ChR2 [15] or pharmacogenetic hM3Dq [16], both resulting in hyperphagia. Furthermore, in some cases pharmacogenetics can prove more effective, as light cannot reach all AAV infected neurons, whereas systemic injection of a chemical actuator easily reaches the targeted area through circulation. Nevertheless, a specific activation of single neuronal circuits is not possible with techniques other than optogenetics. Its extraordinarily high spatiotemporal resolution allowed neurodegenerative disorders other than Parkinson's to be explored with more accuracy [17]. An example includes Alzheimer's disease modeled on mice [5], as through optogenetics a higher temporal resolution for analyzing a specific neural circuit's operation was obtained, allowing its cause-effect mechanism to be observed more clearly $[18,19]$. Existing brain stimulation techniques such as the deep brain stimulation (DBS), the transcranial direct current stimulation, and the transcranial magnetic stimulation lack the ability of specific cell type and neural circuit targeting. Optogenetics has overcome this problem and enabled manipulations with high temporal precision to study the mechanisms of neurological disorders and the causal relationships between single cells and pathways.

\section{Novel therapeutic approach. Circuit- centric targeting concepts.}

Optogenetics paves the way for novel therapeutic approaches, in which chemistry is replaced by micro-optoelectronics and the genetic modification of specific cells and in which the modulation of specific neuronal circuits is the central mechanism of action [20]. From a clinical perspective, the application of optogenetics has begun to be used for exploring vision restoration in inherited retinal diseases (IRD) and selective deep-brain stimulation (DBS) in motor diseases such as Parkinson's. [21]. A limitation in the case of IRD stems from the basic mechanisms of action of optogenetics, namely that the opsins used in studies are most commonly type I (microbial) ion channels while human rhodopsin is a type II opsin ( $\mathrm{G}$ protein coupled receptor), which causes a 
significant difficulty in implementation [22]. However, in the case of DBS, the additional selectivity of optical stimulation can decrease the number of therapy resistant cases [23]. What is more, in the case of Alzheimer's disease, in current mice models researchers can "retrieve memories" via the opsin-mediated activation of hippocampal cells [5]. It has been proven that the optogenetic induction of longterm potentiation at dentate gyrus engram cells restores both spine density and long-term memory. As a result, a new potential method for treating memory loss in the early stage of Alzheimer's disease has been developed. Other papers show that optogenetic treatment can be potentially used to modulate pain signals in the central nervous system [24]. The first demonstration of optogenetic modulation of pain was using channelrhodopsin expression in Nav1.8expressing nociceptors in lamina I and II of the dorsal horn of the spinal cord in mice [25]. In this model, an external light source was able to induce a nocifensive response in the animal. In further studies, the optogenetic silencing of Nav1.8-positive afferent fibers proved to alleviate inflammatory and neuropathic pain [26]. This idea is especially important as an increasing number of people experiences chronic pain and all other forms of treatment, such as painkillers, induce drug dependence or other side effects in such patients [27]. Optogenetic modulation of specific nociceptors may prove to revolutionize the domain of chronic pain treatment.

Furthermore, thanks to optogenetics it is possible to control the activity of enteric neurons, providing a new strategy for treating enteric nervous system (ENS) diseases [6] which are especially difficult to target by traditional methods. The photoactivation of cultured smooth muscle cells using enteric neurons containing SYN-ChR2-eYFP (microbial channelrhodopsin-2) was possible in an in vitro human embryonic stem cell line [28]. This development may in the future become a promising approach to treat ENS neuropathies. Although viral vectors that could deliver channelrhodopsin to inhibitory motor neurons in the mouse colon have not yet been developed, it is only a question of time when optogenetic actuators will be delivered to specific enteric neurons. Thanks to the translational properties of interspecies optogenetics usage, such methods could be easily applied in patients suffering from intestinal motility disorders. However, there are several issues which need to be overcome in order to translate animal-based studies into potential therapies. The main problems are light sources that would not cause damage to surrounding tissue and differences in the expression of microbial opsin genes in certain groups of cells. With the latter limitation, even cautious light usage would induce different effects in certain cells [29]. As a result of these obstacles, optogenetics will not be translatable to human applications in the nearest future. However, an intermediate step could be novel deep brain stimulation (DBS) protocols that emulate successful optogenetic approaches in animal models [30].
Even though optogenetics seems to be a promising method in treatment strategies not only for central nervous system diseases but also for other disorders, it still requires further development.

\section{Global revolution and complicated fu- ture}

Optogenetics began a global revolution. This revolution led to numerous papers being published on the topic, with researchers viewing the method as very promising. Optogenetics has created a possibility to develop improved techniques for therapy and understand the pathophysiology of many diseases better. What is more, the application of optogenetics is not only limited to the nervous system, as methods applied in neurons can also be used to generate a therapeutic effect in other tissues. Already in 2014, Park et. al demonstrated that in ChR2-sensitised ventricular myocytes, action potentials can be easily regulated using certain light stimuli [31]. Similarly, optogenetics is also becoming increasingly applied in developmental studies, most notably as a method for controlling protein localization during embryogenesis [32]. These examples prove that the innovative method not only created space for major developments in neuroscience-related areas, but also many others. However, with all these achievements come significant problems. As previously mentioned, differences in opsin expression in different types of cells and a difficulty with creating non-damaging light sources will most likely slow down the implementation of animal-based studies on patients. What is more, the selective insertion of opsin containing genes is another issue that has not yet been dealt with. Furthermore, when compared to pharmacogenetics, optogenetic mechanisms allow very short-term control of neuronal activity and cannot target a broad area [33], which may diminish the significance of optogenetic-based solutions for patients with chronic issues. For optogenetics to be used in clinics, several years of further studies and new technological achievements are required. Until then, this innovative method can only be used to understand the physiology of, not to treat, certain diseases.

\section{Conclusion}

In conclusion, optogenetics allowed innovative studies based on cell-type specificity and single-cell electrical and biochemical modulation. Furthermore, the development of optogenetic methods allowed for an integrated study of the pathophysiology of many neurological disorders. Moreover, ongoing studies which are based on cell-specific modulation using opsins show that in the near future, this method could be used to alleviate pain or deal with memory loss. Finally, it is important to mention that optogenetics began a biotechnological revolution, which may in the future aid to overcome a variety of issues which prevent develop- 
ment of clinical applications ranging across all scientific fields.

\section{References}

[1] Boyden E et al. Millisecond-Timescale, Genetically Targeted Optical Control of Neural Activity. Nat Neurosci. 2005;8, 1263-1268. doi:10.1038/nn1525.

[2] Pastrana E. Primer on Optogenetics: "Optogenetics: Controlling cell function with light". Nat Methods. 2010; 8 (1): 24-25. doi:10.1038/nmeth.f.323.

[3] Deisseroth K. "Optogenetics". Nat Methods.2011;8(1): 26-9.doi:10.1038/n meth.f.324.

[4] Reiner A, Isacoff EY. The Brain Prize 2013: the optogenetics revolution. Trends Neurosci. 2013;36,10, 557 - 560. doi: 10.1016/j.tins.2013.08.005.

[5] Roy D, Arons A, Mitchell T et al. Memory retrieval by activating engram cells in mouse models of early Alzheimer's disease. Nature. 2016;531, 508512. doi:10.1038/nature 17172.

[6] Wang W, Optogenetic manipulation of ENS The brain in the gut. Life Sci. 2018;192,1920.doi:10.1016/j.lfs.2017.11.010.

[7] Grote M, Engelhard M, Hegemann P. Of ion pumps, sensors and channels. Perspectives on microbial rhodopsins between science and history. Biochimica et Biophysica Acta (BBA) - Bioenergetics. 2014;1837(5):533-545. doi:10.1016/j.bbabio.2013.08.006.

[8] Feuda R, Hamilton SC, McInerney JO, Pisani D. Metazoan opsin evolution reveals a simple route to animal vision. Proc Natl Acad Sci USA. 2012;109(46):1886818872. doi:10.1073/pnas.1204609109.

[9] Kim KK, Adhikari A, Deisseroth K. Integration of Optogenetics With Complementary Methodologies in Systems Neuroscience. Nat Rev Neurosci. 2017;18(4),222-235. doi:10.1038/nrn.2017.15.

[10] Airan RD, Thompson KR, Fenno LE, et al. Temporally precise in vivo control of intracellular signaling. Nature. 2009;7241(458):1025-1029. doi: 10.1038/nature07926.

[11] Hughes RM, Lawrence DS. Optogenetic engineering: light-directed cell motility. Angew Chem Int Ed Engl. 2014 6;53(41):10904-7. doi: 10.1002/anie.201404198.

[12] Haar LL, Lawrence DS, Hughes RM. Optogenetic perturbation of the biochemical pathways that control cell behavior. Methods Enzymol. 2019;622:309-328. doi:10.1016/bs.mie.2019.02.020.

[13] Dewson G, Kluck RM. Mechanisms by which Bak and Bax permeabilise mitochondria during apoptosis. J Cell Sci. 2009;122(16):2801-2808. doi:10.1242/jcs.038166.
[14] Savoie K. The Optogenetics Revolution. Columbia Medicine. 2014. columbiamedicinemagazine.org/features/spring2014/optogenetics-revolution. Accessed on 19.08.2020.

[15] Aponte Y, Atasoy D, Sternson SM. AGRP neurons are sufficient to orchestrate feeding behavior rapidly and without training. Nat Neurosci. 2011;14(3):351-5. doi: 10.1038/nn.2739.

[16] Krashes MJ, Koda S, Ye C, Rogan SC, Adams AC, Cusher DS, Maratos-Flier E, Roth BL, Lowell BB. Rapid, reversible activation of AgRP neurons drives feeding behavior in mice. J Clin Invest. 2011;121(4):1424-8. doi: 10.1172/JCI46229.

[17] Jiang J, Cui H, Rahmouni K. Optogenetics and pharmacogenetics: principles and applications. Am J Physiol Regul Integr Comp Physiol. 2017;313(6):633-645. doi:10.1152/ajpregu.00091.2017.

[18] Zhang F, Aravanis AM, Adamantidis A et al. Circuitbreakers: optical technologies for probing neural signals and systems. Nat Rev Neurosci. 2007;8(8):577581.doi:10.1038/nrn2192.

[19] Fenno L, Yizhar O, Deisseroth K. The development and application of optogenetics. Annu Rev Neurosci. 2011;34:389-412. doi:10.1146/annurev-neuro-061010113817.

[20] Song C, Knöpfel T. Optogenetics enlightens neuroscience drug discovery. Nat Rev Drug Discov. 2016;15, 97-109. doi:10.1038/nrd.2015.15.

[21] Towne C, Thompson KR. Overview on Research and Clinical Applications of Optogenetics. Curr Protoc Pharmacol. 2016;75:11.19.1-11.19.21. doi:10.1002/cpph.13.

[22] Simunovic MP, Shen W, Lin JY, Protti DA, Lisowski L, Gillies MC. Optogenetic approaches to vision restoration. Experimental Eye Research. 2019;178:1526.doi:10.1016/j.exer.2018.09.003.

[23] Delbeke J, Hoffman L, Mols K, Braeken D, Prodanov D. And Then There Was Light: Perspectives of Optogenetics for Deep Brain Stimulation and Neuromodulation. Front Neurosci. 2017;11:663. doi:10.3389/fnins.2017.00663.

[24] Xie Y, Wang J, Bonin RP. Optogenetic exploration and modulation of pain processing. Experimental Neurology. 2018;306:117-121. doi: 10.1016/j.expneurol.2018.05.003.

[25] Daou I, Tuttle AH, Longo G, Wieskopf JS, Bonin RP, Ase AR, Wood JN, De Koninck Y, Ribeiro-daSilva A, Mogil JS, Séguéla P. Remote optogenetic activation and sensitization of pain pathways in freely moving mice. J Neurosci. 2013;33(47):18631-40. doi: 10.1523/JNEUROSCI.2424-13.2013. 
[26] Daou I, Beaudry H, Ase AR, et al. Optogenetic Silencing of Nav1.8-Positive Afferents Alleviates Inflammatory and Neuropathic Pain. eNeuro. 2016;3(1):ENEURO.0140-15.2016.

[27] Ives J. Current and Future Applications of Optogenetics. News Medical. 2018. news-medical.net/lifesciences/Current-and-Future-Applications-ofOptogenetics.aspx.

[28] Fattahi F, Steinbeck JA, Kriks S, et al. Deriving human ENS lineages for cell therapy and drug discovery in Hirschsprung disease. Nature. 2016;531(7592):105109. doi:10.1038/nature16951.

[29] Delbeke J, Hoffman L, Mols K, et al. And Then There Was Light: Perspectives of Optogenetics for Deep Brain Stimulation and Neuromodulation. Front Neurosci. 2017;11:663. doi:10.3389/fnins.2017.00663.

[30] Lüscher C, Pollak P. Optogenetically inspired deep brain stimulation: linking basic with clinical research. Swiss Med Wkly. 2016;146:w14278. doi:10.4414/smw.2016.14278.

[31] Park SA, Lee SR, Tung L, et al. Optical mapping of optogenetically shaped cardiac action potentials. Sci Rep. 2014;4:6125. doi:10.1038/srep06125.

[32] Uchida A, Yajima M. An optogenetic approach to control protein localization during embryogenesis of the sea urchin. Dev Biol. 2018;441(1):19-30. doi:10.1016/j.ydbio.2018.06.015.

[33] Jiang J, Cui H, Rahmouni K. Optogenetics and pharmacogenetics: principles and applications. Am J Physiol Regul Integr Comp Physiol. 2017;313(6):633-645. doi:10.1152/ajpregu.00091.2017. 